\title{
Pityriasis Rosea Associated With COVID-19 Vaccination: A Common Rash Following Administration of a Novel Vaccine
}

\author{
Brittany Valk, DO; Brett Bender, DO
}

\section{PRACTICE POINTS}

- Clinicians should be aware of the association between COVID-19 vaccination and the development of pityriasis rosea.

- Pityriasis rosea has been linked to reactivation of human herpesvirus 6 and human herpesvirus 7 and has been reported following administration of the influenza and human papillomavirus vaccines.

- Pityriasis rosea is a self-limited, cutaneous eruption that resolves within 6 to 8 weeks, and patients should be educated on the benign nature of this condition.

Pityriasis rosea is a common, self-limited, papulosquamous eruption, classically presenting along the Langer lines of the skin. We report a clinically typical case of pityriasis rosea that developed following COVID-19 vaccination. As the novel COVID-19 vaccine becomes more widely available during the ongoing COVID-19 pandemic, it is imperative for both patients and clinicians to be aware of its association with the onset of this benign cutaneous eruption.

Cutis. 2021;108:317-318.

$\square$ ityriasis rosea is a papulosquamous eruption that favors the trunk and proximal extremities. It occurs most commonly in adolescents and young adults. ${ }^{1}$ The rash typically presents with a solitary lesion, known as a"herald patch," which is followed by a scaly erythematous eruption along the cleavage lines of the skin. The condition is self-limited and often resolves in 6 to 8 weeks. Recent evidence suggests that viral reactivation of human herpesvirus 6 and human herpesvirus 7 may play a role in the development of skin lesions. ${ }^{2}$ Pityriasis rosea also has been reported following the administration of new medications and vaccinations. ${ }^{1-3}$ We report a case of a 30 -year-old woman who developed pityriasis rosea 3 days after receiving the second dose of the COVID-19 vaccine.

\section{Case Report}

A 30-year-old woman presented to the dermatology office for evaluation of a rash on the trunk and upper extremities that had been present for 5 days. She reported an initial solitary lesion on the left upper back, subsequently followed by the appearance of a mildly pruritic rash on the trunk and upper extremities. The rash first appeared 3 days after she received the second dose of the Pfizer-BioNTech COVID-19 vaccine. She was otherwise asymptomatic after vaccination and denied fever, chills, headache, and myalgia. She denied any rash following her first dose of the COVID-19 vaccine, history of known COVID-19 infection or exposures, or new medications. Notably, the patient worked in health care.

Physical examination revealed a 2-cm, erythematous, thin, scaly plaque over the left side of the upper back (Figure, A). Erythematous, scaly, thin papules of varying sizes were distributed along the cleavage lines of the trunk and upper extremities (Figure, B). No biopsy was performed because of the classic clinical presentation of

From the Department of Dermatology, Beaumont Hospital Farmington Hills, Michigan.

The authors report no conflict of interest.

Correspondence: Brittany Valk, DO, Department of Dermatology, Graduate Medical Education, Beaumont Hospital, 28050 Grand River Ave,

Farmington Hills, Ml 48338 (brittanyvalk@gmail.com).

doi: 10.12788 /cutis.0411 
A, Classic pityriasis rosea "herald patch" on the left side of the upper back. B, Erythematous scaly papules along cleavage lines of the skin on the trunk.
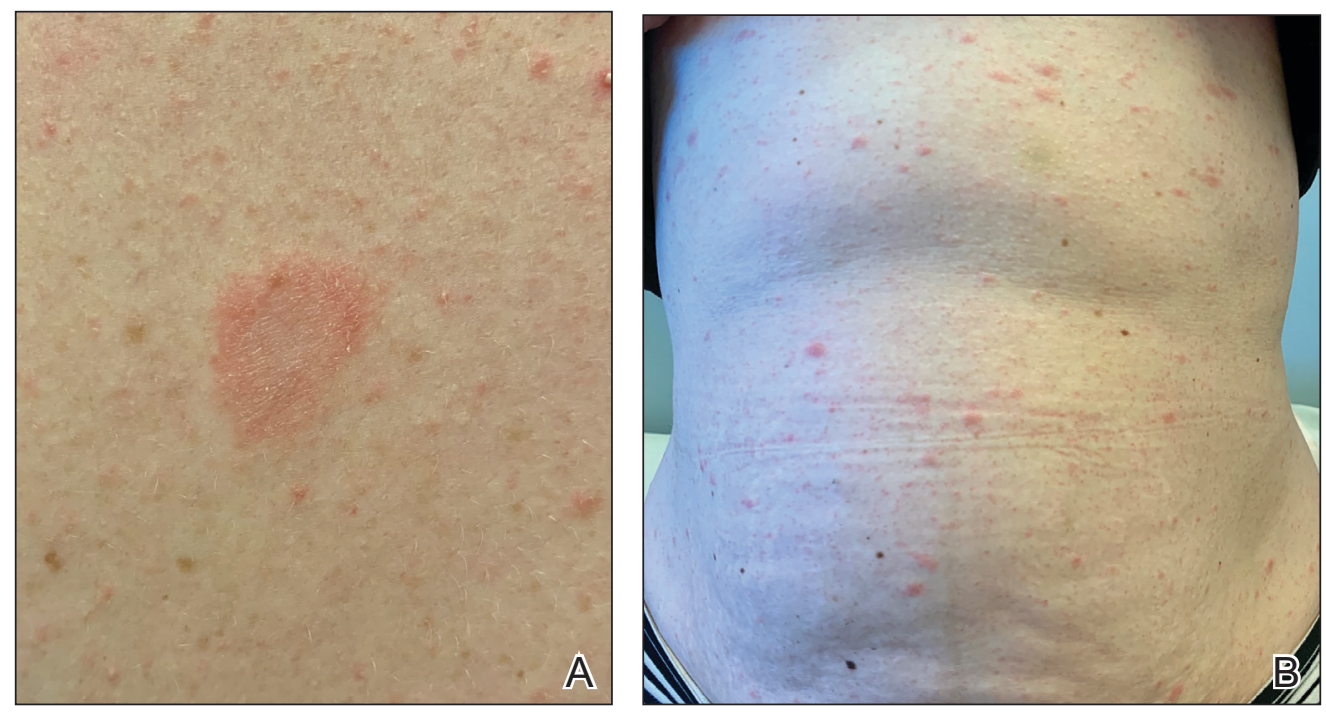

this self-limited condition and the patient's history of hypertrophic scarring. No additional laboratory workup was performed. She was prescribed triamcinolone cream $0.1 \%$ as needed for pruritus and was reassured about the benign nature of this cutaneous eruption.

\section{Comment}

A broad spectrum of cutaneous manifestations has been reported in association with acute COVID-19 infection, including a papulovesicular rash, perniolike eruptions, urticaria, livedo reticularis, and petechiae. ${ }^{4}$ Several cases of pityriasis rosea in association with acute COVID-19 infection also have been reported..$^{5}$ COVID-19 infection has been linked to reactivation of the herpesvirus, which may explain the connection between acute COVID-19 infection and the development of pityriasis rosea. ${ }^{6}$ Pityriasis rosea associated with administration of the COVID-19 vaccine is a rare complication with few reports in the literature. ${ }^{7}$ Similar to our patient, there are reports of pityriasis rosea developing after the second dose of the vaccine, with some patients reporting a reactivation of skin lesions. ${ }^{8}$ There is a paucity of reports describing pityriasis rosea associated with the influenza vaccine, hepatitis B vaccine, and human papillomavirus vaccine. ${ }^{3}$ In such cases, the onset of skin lesions was thought to be related to vaccine-induced stimulation of the immune system or a component of the vaccine.

\section{Conclusion}

We presented a unique case of pityriasis rosea following COVID-19 vaccination. Because additional laboratory workup and a skin biopsy were not performed, we are unable to infer causation. However, the classic clinical presentation, rash development within 3 days of vaccination, and prior reports of vaccine-associated pityriasis rosea strengthen the aforementioned association. We hope this case adds to the growing understanding of the novel COVID-19 vaccine. As more individuals become vaccinated, both clinicians and patients should be aware of this benign cutaneous eruption that can develop following COVID-19 vaccination.

\section{REFERENCES}

1. Papakostas D, Stavropoulos PG, Papafragkaki D, et al. An atypical case of pityriasis rosea gigantea after influenza vaccination. Case Rep Dermatol. 2014;6:119-123.

2. Chen FJ, Chian $\mathrm{CP}$, Chen YF, et al. Pityriasis rosea following influenza (H1N1) vaccination. J Chin Med Assoc. 2011;74:280-282.

3. Li A, Li P, LiY, et al. Recurrent pityriasis rosea: a case report. Hum Vaccin Immunother. 2018;4:1024-1026.

4. Ng SM. Prolonged dermatological manifestation 4 weeks following recovery of COVID-19 in a child. BMJ Case Rep. 2020;13:e237056. doi:10.1136/bcr-2020-237056

5. Johansen M, Chisolm SS, Aspey LD, et al. Pityriasis rosea in otherwise asymptomatic confirmed COVID-19-positive patients: a report of 2 cases. JAAD Case Rep. 2021;7:93-94.

6. Dursun R, Temiz SA. The clinics of HHV-6 infection in COVID-19 pandemic: pityriasis rosea and Kawasaki disease. Dermatol Ther. 2020;33:e13730. doi:10.1111/dth.13730

7. Leerunyakul K, Pakornphadungsit K, Suchonwanit P. Case report: pityriasis rosea-like eruption following COVID-19 vaccination [published online September 7, 2021]. Front Med. doi:10.3389 /fmed.2021.752443

8. Marcantonio-Santa Cruz OY, Vidal-Navarro A, Pesqué D, et al. Pityriasis rosea developing after COVID-19 vaccination. J Eur Acad Dermatol Venereol. 2021;35:E721-E722. doi:10.1111/jdv.17498 